\title{
EPIDEMIOLOGY AND PATHOPHYSIOLOGICAL MECHANISMS INVOLVED IN ALTERATION OF GLOMERULAR FUNCTION IN DIABETIC PATIENTS
}

\author{
Marcel STOITA ${ }^{1}$, Areha Abid ${ }^{2}$, Cosmin VESA ${ }^{1 \star} \bowtie$, Raluca A. CORB ARON ${ }^{1}$, Timea C. GHITEA ${ }^{1}$, \\ Gabriela ANGELESCU ${ }^{3}$, Amorin POPA ${ }^{1}$
}

${ }^{1}$ Faculty of Medicine and Pharmacy, University of Oradea, Oradea, Romania

${ }^{2}$ Faculty of Agricultural and Food Sciences, University of Debrecen, Debrecen, Hungary

3 "Carol Davila" University of Medicine and Pharmacy, Internal Medicine Clinic, Clinical Emergency Hospital of Ilfov County, Bucharest, Romania

Received 02 Febr 2021, Accepted 28 Febr 2021

hitps://doi.org/10.31688/ABMU.2021.56.1.12

\begin{abstract}
Chronic kidney disease of diabetic cause is a highly prevalent complication in diabetes mellitus patients. Complex phenotypes of the disease start to be recognized, such as albuminuria (micro- or macroalbuminuria) and non-albuminuric phenotype reduced glomerular filtration rate $<60 \mathrm{~mL} / \mathrm{min} / 1.73 \mathrm{~m}^{2}$. Numerous pathogenic pathways contribute to the development of this disorder, as follows: the alterations of glucose metabolism with excess degradation of glucose on alternative pathways, hemodynamic changes, glomerulosclerosis, inflammation. As this review demonstrates once more, all these pathophysiological modifications can be linked to hyperglycaemia. Both glomerular and tubular interstitial lesions occur and contribute to disease progression. Adequate glycaemic control and an early diagnosis of diabetes mellitus are key measures in preventing this complication.
\end{abstract}

Keywords: epidemiology, diabetes, chronic kidney disease, glomerular function, insulin.
Résumé

Épidémiologie et mécanismes pathophysiologiques impliqués dans la modification de la fonction glomérulaire chez les patients diabétiques

La maladie rénale chronique de cause diabétique est une complication très répandue chez les patients diabétiques. Des phénotypes complexes de la maladie commencent à être reconnus, tels que l'albuminurie (micro-ou macro-albuminurie) et le phénotype non albuminurique réduit du taux de filtration glomérulaire $<60 \mathrm{~mL} / \mathrm{min} / 1,73 \mathrm{~m}^{2}$. De nombreuses voies pathogènes contribuent au développement de ce trouble, comme : les altérations du métabolisme du glucose avec une dégradation excessive du glucose sur des voies alternatives, les modifications hémodynamiques, la glomérulosclérose, l'inflammation. Comme cette revue le démontre une fois de plus, toutes ces modifications physiopathologiques peuvent être liées à une hyperglycémie. Des lésions de l'interstice glomérulaire et tubulaire se produisent et contribuent à la progression 


\section{Abbreviations list}

ACEI - angiotensin converting enzyme inhibitors

AGE - advanced enzymatic glycosylation

ARB - angiotensin receptor blockers

CKD - chronic kidney disease

DAG - diacylglycerol

DCCT- Diabetes Control and Complications Trial

DHAP - dihydroxyacetone phosphate DHAP

DM - diabetes mellitus

GFR - glomerular filtration rate

NF- $\kappa \mathrm{B}$ - nuclear factor kappa activator of light chains

of activated $B$ cells

NHANES - National Health and Nutrition

Examination Survey

PDGF-B - factor B subunit platelet-derived growth fac-

tor

PKC - protein kinase C

SGLT-2 - sodium-glucose type 2

TGF- $\beta 1$ - transforming growth factor- $\beta 1$

TNF- $\alpha$ - tumour necrosis factor- $\alpha$

UKPDS - United Kingdom Prospective Diabetes

Study

VEGF - endothelial vascular growth factor

\section{Introduction. General aspects REGARDing CHRONIC KIDNEY DISEASE OF DIABETIC CAUSE}

Chronic kidney disease (CKD) of diabetic cause means loss of renal function as a consequence of diabetes mellitus (DM), being defined as progressive impairment of renal function in a diabetic patient that can be documented by progressive albuminuria, decreased glomerular filtration rate (GFR), increased systolic blood pressure and/or diastolic blood pressure and the occurrence over time of important macrovascular complications such as acute coronary event or stroke ${ }^{1,2}$. CKD of diabetic cause is a leading cause of disease burden in patients with diabetes, considered in developed countries as the most important cause of chronic end-stage renal disease, with need for haemodialysis or renal transplant ${ }^{1,3}$. In type $1 \mathrm{DM}$ patients, CKD generally is identified after about 10 years of evolution of diabetes, while in type 2 DM patients, CKD can already be identified in 3\% of patients at the time of diagnosis ${ }^{1}$. The importance of the permanent epidemiological, pathophysiological, diagnostic and treatment research of CKD of diabetic cause lies in its high prevalence, of over 40\% in patients from the United States of America (USA) and the enormous costs it generates on the healthcare systems of all countries ${ }^{4}$, being estimated that in the USA, in 2011, the costs for patients with DM and CKD were $\$ 25$ billion (1). CKD of diabetic cause is an ongoing challenge for researchers in diabetes de la maladie. Un contrôle glycémique adéquat et un diagnostic précoce du diabète sucré sont des mesures clés pour prévenir cette complication.

Mots-clés: épidémiologie, diabète, maladie rénale chronique, fonction glomérulaire, insuline.

and kidney disease. If only recently the albuminuria was considered absolutely necessary for the diagnosis of diabetic kidney disease, today there are more and more data about two phenotypes, an albuminuric one, characterized by increased urinary excretion of albumin, and a non-albuminuric one, characterized by decreased GFR ${ }^{5}$. Also, in a patient with diabetes there may be certain pathologies that produce CKD independent of diabetes ${ }^{2}$. For the diagnosis of CKD, the GFR should be calculated on the ground of serum creatinine using a validated formula, preferably the CKD-EPI equation ${ }^{1,2}$. To identify a possible rapid decrease in the GFR, this should be repeated at least annually over a period of $3-5$ years ${ }^{1,2}$.

The diagnosis of CKD of diabetic cause is a clinical one, based on the presence of persistent albuminuria (micro- or macroalbuminuria) and/or persistent low GFR $\left(<60 \mathrm{~mL} / \mathrm{min} / 1.73 \mathrm{~m}^{2}\right)$, which corresponds to the fact that the absence of albuminuria is common in patients with renal dysfunction. Renal biopsy is indicated in atypical presentations that suggest the presence of other renal diseases ${ }^{5}$. The known phenotypes of CKD of diabetic cause are depicted in Figure 1.

The purpose of our review is to highlight the most important mechanisms involved in the pathogenesis of CKD of diabetic cause, and to present several epidemiological aspects related to it, emphasizing the need for permanent research in this field, given the growing prevalence of the disease. Bibliography 


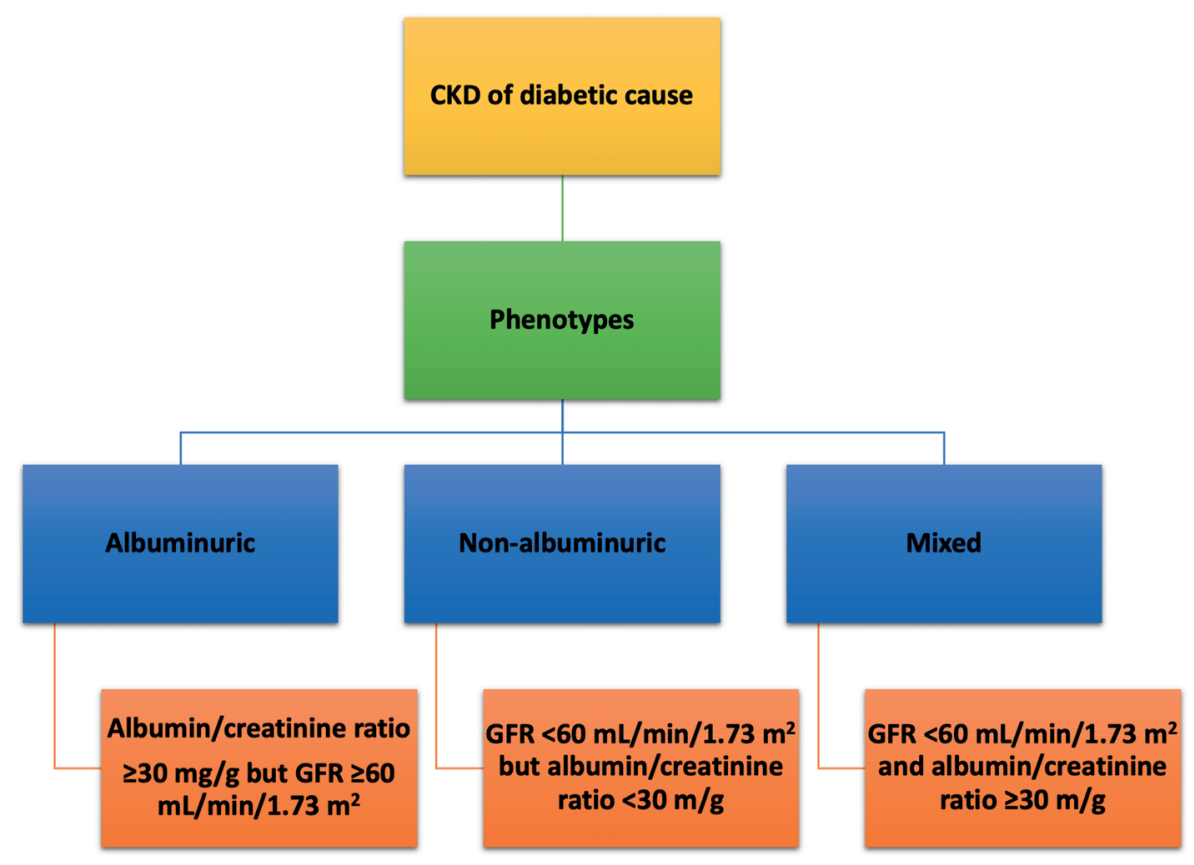

Figure 1. Phenotypes of chronic kidney disease (CKD) of diabetic cause

has been gathered using PUBMED database, using key words related to the main concepts presented in this paper (CKD and DM).

\section{Epidemiology of CKD dUe to diabetes}

Patients with diabetes are about two times more likely to suffer from CKD than people who do not have diabetes ${ }^{6}$. In the National Health and Nutrition Examination Survey (NHANES), in patients evaluated from 2009 to 2014, the prevalence of CKD in this category of patients was $26.2 \%$, the diagnostic criterion being the albuminuria presence ${ }^{6}$. In patients over 65 years of age, the prevalence of low GFR was higher than in young patients, while albuminuria had comparable prevalence in both categories. The prevalence of CKD in patients with diabetes in 2008 in Shanghai, China was $33.5 \%$. In Singapore, the prevalence of CKD in patients with diabetes was $53 \%$, in $21 \%$ of patients a GFR $<60 \mathrm{~mL} / \mathrm{min} / 1.73$ $\mathrm{m}^{2}$ was identified, $48 \%$ had albuminuria $\geq 30 \mathrm{mg} / \mathrm{g}$, and $28 \%$ had diabetic retinopathy? ${ }^{7}$. The parameters that were associated with diabetic kidney disease were the presence of diabetic neuropathy, hypertension, hypertriglyceridemia, increased BMI, increased duration of diabetes, $\mathrm{HbA} 1 \mathrm{c} \geq 8 \%$ and the presence of cardiovascular disease ${ }^{8}$. In Europe, epidemiological data reveal that the occurrence of CKD in this category of patients is 2 to 5 times higher in patients with diabetes than in the nondiabetic population. The prevalence is different, depending on the country, being $14.1 \%$ in the Netherlands, $26.3 \%$ in Norway, $39.7 \%$ in Germany and 22.1\% in Spain ${ }^{8}$. Normal albumin excretion is common in patients with CKD and diabetes. Therefore, we note that the epidemiological studies studied above include in the category of patients with CKD both patients with albuminuria and those with low GFR. In the 1998-2004 NHANES study, $30 \%$ of participants with type $2 \mathrm{DM}$ over 40 years of age or older and with low GFR did not have albuminuria or diabetic retinopathy'.

The United Kingdom Prospective Diabetes Study (UKPDS) study reported that $38 \%$ of newly diagnosed patients with diabetes had albuminuria over a 15-year follow-up period, $29 \%$ had reduced GFR (the renal function was estimated by the Cockroft-Gault equation $<60 \mathrm{~mL} / \mathrm{min} / 1.73 \mathrm{~m}^{2}$ ) or more than two times the plasma creatinine, and $14 \%$ developed as well albuminuria and low GFR ${ }^{10}$. In the Diabetes Control and Complications Trial (DCCT) study, moderate albuminuria cumulative incidence (urinary albumin/creatinine ratio between $30-300 \mathrm{mg} / \mathrm{g}$ ) was $14 \%$ (at 10 years), 33\% (at 20 years) and 38\% (at 30 years) of type $1 \mathrm{DM}$ duration, in people with conventional hypoglycaemic treatment (mean $\mathrm{HbAlc}$ value of $9.6 \%$ ). The incidence of albuminuria was lower, of $10 \%, 21 \%$ and $25 \%$ in patients with intensive treatment of hyperglycaemia ${ }^{11}$. At 18 years from the end of the DCCT study, those in the diabetes intensive care group had a 57\% lower risk of moderate albuminuria and an $84 \%$ lower risk of severe albuminuria (albumin/creatinine ratio $\geq 300 \mathrm{mg} / \mathrm{g}$ ) compared to those who did not receive intensive treatment, which suggests a lasting benefit of intensive treatment of 
hyperglycaemia to prevent the development of CKD of diabetic cause ${ }^{12}$. Patients with CKD of diabetic cause have a much higher risk of premature mortality compared to the general population ${ }^{13}$. According to epidemiological data, in the adult population of the USA, the cumulative mortality of any cause at 10 years standardized by age was $7.7 \%$ in people without $\mathrm{DM}$ and without $\mathrm{CKD}$ (albumin/creatinine ratio $\geq 30$ $\mathrm{mg} / \mathrm{g}$ in urine and/or GFR $\leq 60 \mathrm{~mL} / \mathrm{min} / 1.73 \mathrm{~m}^{2}$ ), $11.5 \%$ in those with DM without CKD and $31.1 \%$ in those with $\mathrm{DM}$ and $\mathrm{CKD}^{13}$. In another group of patients (representing $16 \%$ of all patients with type 1 DM in Finland) that was included in the FinnDiane study, the diagnosis and severity of kidney disease was a major predictor of mortality from any cause $\mathrm{e}^{14}$. Moderate levels of albuminuria (urinary albumin excretion between $20-200 \mathrm{mg} / \mathrm{min}$ ) were correlated with a 3 times higher risk of mortality compared to the entire population. CKD of diabetic cause has a prevalence in a continuous increase, which corresponds to the overall increase in the prevalence of DM. Data show that globally 1 in 3 patients with DM suffer from $\mathrm{CKD}^{15}$. Of concern is the fact that screening for CKD is performed in about $50 \%$ of patients with diabetes ${ }^{15}$.

\section{Pathophysiology}

The most important pathophysiological mechanism involved in the reduction of glomerular function and the occurrence of CKD of diabetic cause is hyperglycaemia ${ }^{16}$. Hyperglycaemia is obviously followed by the metabolism of excess glucose which activates the following alternative metabolic pathways: the pathway of polyols, the pathway of hexosamines, the formation of advanced glycosylation products (AGE), the activation of protein kinase $\mathrm{C}(\mathrm{PKC})^{16}$. The glucose that is present in excess is converted under the action of aldose reductase into sorbitol, with the consumption of NADPH. Low levels of NADPH block the regeneration of reduced glutathione, an important intracellular antioxidant substance, which will increase the level of oxidative stress ${ }^{17}$. The final product of the fructose pathway appears to have an important nephrotoxic effect, in experimental animal models being associated with albuminuria ${ }^{17}$. By hexosamine pathway the metabolite fructose- 6 -phosphate is converted to glucosamine- 6 -phosphate, the conversion being mediated by the enzyme fructose-6-phosphate aminotransferase. Glucosamine-6-phosphate activates the excess secretion / transcription of inflammatory cytokines, like tumour necrosis factor- $\alpha$ (TNF- $\alpha$ ), transforming growth factor- $\beta 1$ (TGF- $\beta 1$ ), the latter further causing renal hypertrophy and the development of components of the mesangial matrix, two very important mechanisms implied in the development of diabetic kidney disease ${ }^{16,17}$.

AGE products are a result of non-enzymatic glycosylation of proteins as consequence of excess glycaemia with numerous negative metabolic consequences. They appear in the 4th stage of glycolysis. AGEs action both extracellularly and intracellularly ${ }^{18}$. Glycation of extracellular matrix proteins has been demonstrated to occur due to hyperglycaemia at the renal level by altering laminin and IV collagen and increasing glomerular permeability ${ }^{18}$. AGE presence also increases fibronectin and collagen I and IV expression at the glomerular level, which increases the density and expansion of the extracellular matrix ${ }^{19}$. AGE interacts with various cellular receptors, specifically the AGE receptors, which will influence the transcription rate of various pro-inflammatory cytokines including interleukins IL-6 and/or IL-1, factors such as TNF- $\alpha$, TGF- $\beta 1$, endothelial vascular growth factor (VEGF), factor B subunit platelet-derived growth factor (PDGF-B) ${ }^{19}$. Like AGE, PKC activation occurs in the fourth stage of glycolysis. The conversion of glyceraldehyde-3-phosphate to dihydroxyacetone phosphate (DHAP) and eventually to diacylglycerol (DAG) is caused by hyperglycaemia, which is a cofactor of PKC activation. PKC activation causes the overproduction of substances such as prostaglandin $\mathrm{E}$ and nitric oxide ${ }^{18,19}$. Thus, vasodilation will occur in the afferent arteriole of the glomerulus, at the same time angiotensin II causes vasoconstriction in the efferent arteriole of the glomerulus, which will lead to glomerular hyperfiltration. PKC activation also causes the accumulation of fibronectin and type IV collagen, in excess which will lead to thickening of the glomerular basement membrane and extracellular matrix leading to a reduced capacity of plasma filtration ${ }^{19}$.

At the renal level, the glomerulus is the first affected component, after the diagnosis of DM being demonstrated that incipient glomerular lesions occur after an interval of 0-3 years. Pathological lesions specific for CKD of diabetes cause at the glomerular level are well documented in the literature and include podocyte injury, thickening of the glomerular basement membranes, extension of the mesangial matrix and loss of renal fenestration ${ }^{18-20}$. These modifications lead to a marked increase in microvascular permeability and damages of the glomerular filtration barrier which will result in increased albumin excretion. Among these above-mentioned pathological changes, thickening of the glomerular membrane is an early morphologic modification in those suffering of CKD of diabetic cause ${ }^{21}$. Abnormal turnover and changes in the extracellular matrix produced by endothelial cells and podocytes are the causes of the increase of 
glomerular membrane thickness. Thickening of the glomerular basement membrane is considered an early sign of podocyte activation ${ }^{21}$. Mesangial cells proliferation is activated in the presence of excess glucose, they produce excess extracellular matrix, which is the mechanism for glomerulosclerosis. Hyperglycaemia causes non-enzymatic glycation of proteins in the mesangial matrix. This pathological process causes lesions characteristic of CKD of diabetic cause, Kimmelstiel-Wilson nodules ${ }^{20,21}$. These nodules that can be identified microscopically are represented by hyaline pink material surrounded by capillary clumps in the renal glomeruli ${ }^{22}$. The tubulo-interstitial compartment has been largely neglected in CKD of diabetic cause, in contrast to numerous research and studies on the lesions that diabetes causes at the glomerular level. Recently, research has shifted from the glomerular paradigm to tubulopathy in CKD of diabetic cause ${ }^{23}$. As a highlight, tubulo-interstitial changes are now considered a determining factor in impaired renal function with a greater impact than glomerular changes. Renal biopsy specimens showed significant changes in the renal tubules, the largest renal cell population, as well as in the interstitium ${ }^{23}$.

Regarding hemodynamic renal changes, glomerular hyperfiltration is the main mechanism that leads to CKD of diabetic cause ${ }^{24}$. Excess glucose activates an increase of transcription of vasoactive mediators such as nitric oxide, endothelial vascular growth factor, glucagon, insulin-like growth factor 1 and prostaglandin will cause dilation of the afferent arteriole of the glomerulus. On the other hand, there are alterations in renal tubular function that are very severe in the evolution of diabetes. Due to the large amount of glomerular filtered glucose, at the tubular level the reabsorption of glucose and sodium is increased due to the increased expression of sodium-glucose type 2 (SGLT-2) receptor transporter at the level of the proximal convoluted tube of the nephron. Therefore, the amount of sodium ions that arrive at the level of the macula densa of the nephron is low, which will cause the process of vasodilation in the afferent arteriole. At the same time, the increase in angiotensin II production will cause vasoconstriction of the efferent arteriole, which will lead to the appearance of glomerular hypertension and hyperfiltration in the early staged of CKD, a mechanism known to induce glomerular stress and to accelerate the process of glomerular sclerosis ${ }^{24,25}$. Excess glucose, resistance to insulin action in target tissues ${ }^{26}$ and compensatory hyperinsulinemia determine endothelial dysfunction by activating pathological cellular mechanisms like increased oxidative stress, PKC activation and the generation of AGE that activate, all of these will promote the activation of signalling pathways with pro-inflammatory effect. The interaction of mediators produced by endothelial cells is altered. Among these mediators, endothelin-1 is a peptide with important vasoactive proprieties produced by endothelial cells. At the endothelial level, hyperinsulinism increases endothelin production, which will lead to vasoconstriction $^{24,27}$. Endothelin receptor activation generates not only vasoconstriction, but also with podocyte inhibition, increased oxidative stress, increased inflammation and fibrosis ${ }^{24,28}$.

Both ischemia and inflammation are other extremely important mechanisms in the complex physiopathology of CKD of diabetic cause. Glomerular and vascular lesions specific for CKD of diabetic cause are determined by an increase in the production of nitric oxide, which causes an increase in the oxygen demand of the residual nephrons ${ }^{20}$. Excess free radical production and excess hypoxia-induced factor production occur as failure to cover this increased need for oxygen at the glomerular level. Hyperglycaemia interferes with the production of this factor and causes fibrosis ${ }^{23}$.

Experimental data prove that inflammation has a relevant function in the pathophysiology of CKD of diabetic cause. The nuclear factor kappa activator of light chains of activated B cells (NF-KB) represents an important regulator of inflammatory factors implied in the glomerular alteration in CKD of diabetic cause. In diabetic kidney disease, increased TNF- $\beta$ and reactive oxygen species activate NF-kB phosphorylation, which is in relation with increased proteinuria and inflammatory cell infiltration of the renal interstiti$u^{20,21}$. Innate immune system activation is caused by the increase in renal oxidative stress, with consecutive increased secretion of chemokines and/or cell adhesion molecules and molecular patterns correlated with a state of stress ${ }^{22}$. The beginning of the innate renal immune response is done by recruiting macrophages. As hyperglycaemia progresses, AGE and oxidized lipoproteins will cause the immune complexes formation. These immune complexes will be deposited at the renal level and will further amplify the immune response by activating the complement system. At the glomerular level, the activated complement system eases the affiliation of new mast cells and leukocytes ${ }^{18-20}$. As CKD of diabetic cause progresses, the risk of developing renal ischemia increases, which exacerbates kidney inflammation by increasing infiltration of renal neutrophils and macrophages ${ }^{20}$.

Hyperglycaemia and AGE induce renal angiotensin expression in the kidney by increasing oxidative stress and kidney-specific G protein-coupled with the metabolic receptor GPR91 ${ }^{22,23}$. Moreover, increased salt intake, specific for diabetic patients with metabolic syndrome, may decrease the antihypertensive 


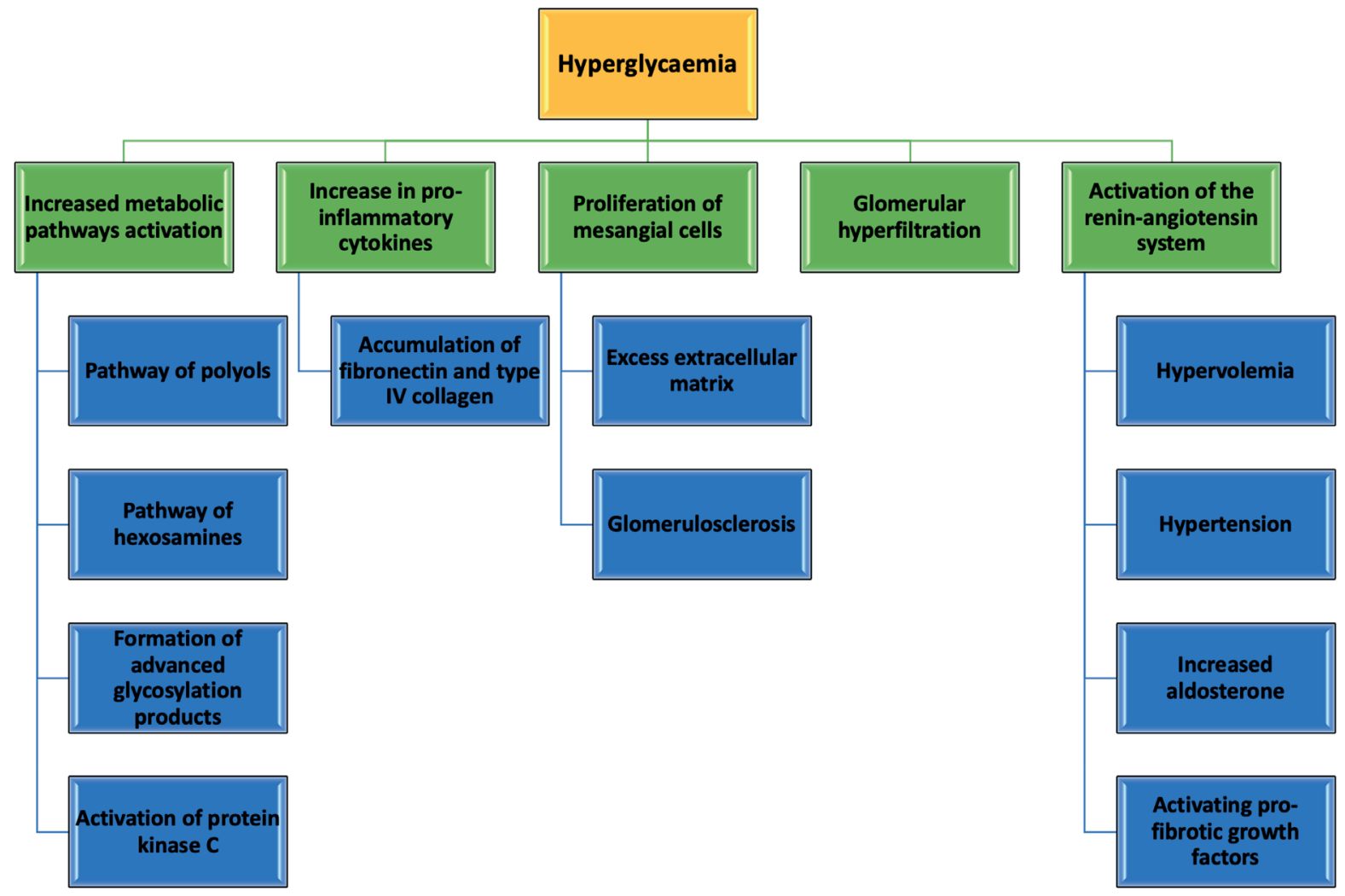

Figure 2. Pathological mechanisms induced by hyperglycaemia in chronic kidney disease (CKD) of diabetic cause

and proteinuria-lowering effect of angiotensin converting enzyme inhibitors (ACEI) and/or angiotensin receptor blockers $(\mathrm{ARB})$ by activating the renin-angiotensin system. Renin-angiotensin plasmatic system activation is one of the most important mechanisms implied in the pathophysiology of CKD of diabetic cause $^{29,30}$. Both TNF- $\beta$ and angiotensin II are mediators involved in the process of renal fibrosis, which can include renal tubule dysfunction and/or their atrophy, reduction in the number of small vessels or hypoxia. Excess aldosterone also plays an important role in CKD of diabetic cause by regulating gene expression and activating pro-fibrotic growth factors (i.e. plasminogen-1 and/or TGF- $\beta$ ), and by increasing renal macrophage infiltration with the development of consecutive fibrosis ${ }^{28}$.

The main aspects that were highlighted in this paper are summarized in Figure 2.

\section{Conclusions}

Hyperglycaemia is the main factor that activates pathogenetic pathways that lead to modifications of renal structure and functioning in CKD of diabetic cause. Complex hemodynamic changes, increased angiotensin production, pathologic modifications in glucose metabolism with generation of toxic products, activation of inflammatory pathways and renal fibrosis are the most important mechanisms involved in CKD of diabetes cause. Our review presents prove that CKD of diabetic cause is a disease of high complexity and without their understanding no advance can be done in its prevention or management.

\section{Author Contributions:}

Conceptualization, M.S, C.M.V, R.A.C.A. and A.P.; resources, M.S. and A.P.; data curation, A.A., R.A.C.A., T.C.G. and A.P.; writing-original draft preparation, M.S and A.P.; writing-review and editing, T.C.G. and C.M.V.; visualization, M.S. and A.A.; supervision, A.P.; project administration, A.P. All authors have read and agreed with the final version of this article.

\section{Compliance with Ethics Requirements:}

"The authors declare no conflict of interest regarding this article"

"No funding for this study"

\section{Acknowledgements:}

None 


\section{References}

1. Tuttle KR, Bakris GL, Bilous RW. Diabetic kidney disease: a report from an ADA Consensus Conference. Diabetes Care. 2014;37(10):2864-2883.

2. Shahbazian H, Rezaii I. Diabetic kidney disease; review of the current knowledge. J Renal Inj Prev. 2013;2(2):73-80.

3. Bailey RA, Wang Y, Zhu V, Rupnow MF. Chronic kidney disease in US adults with type 2 diabetes: An updated national estimate of prevalence based on Kidney Disease: Improving Global Outcomes (KDIGO) staging. BMC Res Notes. 2014; 7: 415.

4. Morgovan C, Cosma S, Valeanu M, et al. An exploratory research of 18 years on the economic burden of diabetes for the Romanian National Health Insurance System. Int $J$ Environ Public Health. 2020;17:4456.

5. Pugliese G, Penno G, Natali A, et al; Italian Diabetes Society and the Italian Society of Nephrology. Diabetic kidney disease: New clinical and therapeutic issues. Joint position statement of the Italian Diabetes Society and the Italian Society of Nephrology on "The natural history of diabetic kidney disease and treatment of hyperglycaemia in patients with type 2 diabetes and impaired renal function". Nutr Metab Cardiovasc Dis. 2019;29(11):1127-1150.

6. Afkarian M, Zelnick LR, Hall YN, et al. Clinical manifestations of kidney disease among US adults with diabetes, 1988-2014. JAMA. 2016;316(6):602-610.

7. Zhou Y, Echouffo-Tcheugui JB, Gu JJ, et al. Prevalence of chronic kidney disease across levels of glycemia among adults in Pudong New Area, Shanghai, China. BMC Nephrol. 2013;14:253.

8. Brück K, Stel VS, Gambaro G, et al. CKD prevalence varies across the European general population. J Am Soc Nephrol. 2016;27(7):2135-2147.

9. Kramer HJ, Nguyen QD, Curhan G, Hsu C-Y. Renal insufficiency in the absence of albuminuria and retinopathy among adults with type 2 diabetes mellitus. JAMA. 2003;289(24):3273-3277

10. Retnakaran R, Cull CA, Thorne KI, Adler AI, Holman $\mathrm{RR}$, Group US. Risk factors for renal dysfunction in type 2 diabetes: U.K. Prospective Diabetes Study 74. Diabetes. 2006;55(6): 1832-1839.

11. Boer IH, Rue TC, Cleary PA, et al. Long-term renal outcomes of patients with type 1 diabetes mellitus and microalbuminuria: an analysis of the diabetes control and complications trial/epidemiology of diabetes interventions and complications cohort. Arch Intern Med. 2011;171(5):412-420.

12. DCCT/EDIC research group. Effect of intensive diabetes treatment on albuminuria in type 1 diabetes: long-term follow-up of the Diabetes Control and Complications Trial and Epidemiology of Diabetes Interventions and Complications study. Lancet Diabetes Endocrinol. 2014;2(10):793-800.

13. Afkarian M, Sachs MC, Kestenbaum B, et al. Kidney disease and increased mortality risk in type 2 diabetes. J Am Soc Nephrol. 2013;24(2):302-308
14. Groop P-H, Thomas MC, Moran JL, et al. The presence and severity of chronic kidney disease predicts all-cause mortality in type 1 diabetes. Diabetes. 2009;58(7):1651-1658

15. Centers for Disease Control and Prevention. National Chronic Kidney Disease Fact Sheet, 2017. Atlanta, GA: US Department of Health and Human Services, Centers for Disease Control and Prevention; 2017.

16. Lanaspa MA, Ishimoto T, Cicerchi C, et al. Endogenous fructose production and fructokinase activation mediate renal injury in diabetic nephropathy. J Am Soc Nephrol. 2014;25(11):2526-2538.

17. Schleicher ED, Weigert C. Role of the hexosamine biosynthetic pathway in diabetic nephropathy. Kidney International Supplements. 2000;77:S13-S18

18. Reidy K, Kang HM, Hostetter T, Susztak K. Molecular mechanisms of diabetic kidney disease. Journal of Clinical Investigation. 2014;124:2333-2340.

19. Fu H, Liu S, Bastacky SI, Wang X, Tian XJ, Zhou D. Diabetic kidney diseases revisited: A new perspective for a new era. Mol Metab. 2019;30:250-263.

20. Lin YC, Chang YH, Yang SY, Wu KD, Chu TS. Update of pathophysiology and management of diabetic kidney disease. J Formos Med Assoc. 2018;117(8):662-675.

21. Tuttle KR. Back to the future: glomerular hyperfiltration and the diabetic kidney. Diabetes. 2017;66(1):14-16.

22. Potenza MA, Gagliardi S, Nacci C, Carratu' MR, Montagnani M. Endothelial dysfunction in diabetes: from mechanisms to therapeutic targets. Curr Med Chem. 2009; 16(1):94-112.

23. de Zeeuw D, Coll B, Andress D, et al. The endothelin antagonist atrasentan lowers residual albuminuria in patients with type 2 diabetic nephropathy. J Am Soc Nephrol. 2014;25(5):1083-93.

24. Vesa, CM, Popa L, Popa A, et al. Current data regarding the relationship between type 2 diabetes mellitus and cardiovascular risk factors. Diagnostics. 2020; 10:314.

25. Gheorghe G, Toth PP, Bungau S, et al. Cardiovascular risk and statin therapy considerations in women. Diagnostics. 2020;10(7):483.

26. Bungau S, Behl T, Tit DM, et al. Interactions between leptin and insulin resistance in patients with prediabetes, with and without NAFLD. Exp Ther Med. 2020;20(6):197.

27. Popa AR, Bungau S, Vesa CM, et al. Evaluating the efficacy of the treatment with benfotiamine and alpha-lipoic acid in distal symmetric painful diabetic polyneuropathy. Rev Chim (Bucharest). 2019;70(9):3108-3114.

28. Popa AR, Fratila O, Rus M, et al. Risk factors for adiposity in the urban population and influence on the prevalence of overweight and obesity. Exp Ther Med. 2020;20: 129-133.

29. He J, Xu Y, Koya D, Kanasaki K. Role of the endothelial-to-mesenchymal transition in renal fibrosis of chronic kidney disease. Clin Exp Nephrol. 2013;17(4):488-97.

30. Ritz E, Tomaschitz A. Aldosterone, a vasculo-toxic agent--novel functions for an old hormone. Nephrol Dial Transplant. 2009;24(8):2302-5. 